\title{
Bioprosthetic heart valve calcification: membrane- mediated events and alkaline phosphatase
}

\author{
F.J. Schoen ${ }^{1}$ and R.J. Levy ${ }^{2}$ \\ 'Department of Pathology, Brigham and Women's Hospital and Harvard Medical School, Boston, MA, \\ and ${ }^{2}$ Department of Pediatrics and Communicable Diseases, University of Michigan, \\ Ann Arbor, MI, USA
}

Calcification-induced stenosis or tearing is the principal cause of failure of clinical heart valve bioprostheses fabricated from glutaraldehyde-pretreated porcine aortic valves and impairs the durability of valves made of glutaraldehyde-pretreated bovine pericardium [1]. Calcification also complicates the use of other medical devices and biomaterials, including valved human aortic homografts, dura mater and fascia lata bioprostheses, trileaflet polymer valves, experimental ventricular assist systems/artificial hearts, contact lenses and intrauterine contraceptive devices [2].

\section{Pathophysiology of bioprosthetic tissue mineralization}

Orthotopic tricuspid or mitral valve replacements or conduit-mounted valves in juvenile sheep or calves calcify extensively in 3-6 months, compared with the several years normally required for calcification of clinical bioprostheses [1,2]. Dramatic acceleration is achieved in subcutaneous (subO) implants of bioprosthetic tissue in young rats, in which levels conparable to those of failed clinical explants (200-225 $\mu \mathrm{g}$ calcium $/ \mathrm{mg}$ tissue) occur in 8 weeks or less [1,2].

Bioprosthetic tissue (BPT) calcification depends on host, implant, and biomechanical factors $[1,2]$. Young age and renal failure potentiate mineralization; the clinically observed age dependence of calcification rate is simulated in the rat subQ model, in which BPT implanted in 8 month old rats accumulate $<15 \%$ that in 3 week old recipients [3]. Moreover, neither non-specific inflammation nor specific immunological responses mediate BPT calcification; tissue reaction to bioprosthetic tissue is a classic non-specific foreign body reaction. Host cell reaction to mineral deposits is minimal, and inflammation induces neither mineral deposition nor resorption. Enclosure of valve cusps in chambers that prevent host cell contact with BPT, but allow free diffusion of extracellular fluid, do not affect mineralization [3]. In addition, implanted BPT calcifies equivalently in immunologically competent 
hosts or congenitally athymic ('nude') mice, that have essentially no T-lymphocyte function [4]. Although BPT likely remains immunogenic despite cross-linking $[25,26]$, a role of the immune response in mineralization remains speculative.

Aldehyde cross-linking is a prerequisite for calcification of BPT in rat subQ implants; non-preserved cusps do not mineralize [5]. The kinetics, extent, and morphology of calcification of glutaraldehyde-treated porcine aortic valve and bovine pericardium are similar [6,7]. These data suggest that the fundamental mechanisms of bioprosthetic tissue mineralization depend on aldehyde pretreatment-induced biochemical modifications of implant structural components.

Calcification of bioprosthetic valves is potentiated in areas of leaflet flexion, where deformations are maximal (i.e., cuspal commissures and bases). Nevertheless, subQ implants calcify with a morphology analogous to that observed in clinical and experimental circulatory implants. Thus, dynamic stress promotes, but is not a prerequisite for, BPT calcification. This suggests that metabolic and iruplant factors alone are both necessary an $\mathrm{I}$ sufficient.

The earliest mineral deposits in both clinical and experimental BPT are localized to transplanted connective tissue cells; collagen involvement occurs subsequently $[3,6,7]$. Cellular mineralization is hypothesized to result from glutaraldehyde-induced cellular 'devitalization' and resulting disruption of cellular calcium regulation [7], that normally maintains intracellular free calcium concentrations at low levels (approximately $10^{-7} \mathrm{M}$ ), although extracellular free calcium is higher (approximately $10^{-3} \mathrm{M}$ ). The 10,000 fold gradient across the plasma membrane is maintained by energy-requiring metabolic processes, such as plasma membranebound $\mathrm{Ca}^{2+}$-ATPase, which uses the energy of ATP hydrolysis to pump $\mathrm{Ca}^{2+}$ out, and intracellular binding by soluble cytosolic or membrane-bound proteins. We have hypothesized that passive calcium entry occurs unimpeded in cells modified by aldehyde cross-linking, since such cells have dysfunctional mechanisms for calcium removal. In this model, calcium influx would contribute to mineral formation by reacting with bound phosphorus as phospholipids in organellar and plasma membranes. Studies using electron energy loss spectroscopy (EELS) formation of calcium phosphate deposits within two days at focally high concentrations of intracellular phosphorus in unimplanted, glutaraldehyde-preserved porcine aortic valve and bovine pericardium [8]. Early crystal nuclei progressively accumulate additional mineral, eventuating in macroscopic calcific deposits. Whether collagen deposits are related to contiguous cell-oriented mineral deposition or arise independently is unknown. However, calcification of cells and collagen could occur independently, since calcification of aldehyde-cross-linked Type I collagen sponges occurs without associated cell-oriented calcification [9].

'Calcific diseases' (e.g., degenerative calcific aortic stenosis, atherosclerosis) and physiological calcification of skeletal and dental tissues share important features [2]. In particular, both pathologic and physiologic mineralization yield crystal deposits associated with cells and collagen. Nearly all forms of calcification occur by crystal formation on cell membranes, usually in the form of extracellular vesicles, often called 'matrix vesicles', that have both a high concentration of calciumbinding acidic phospholipids within their membranes, as well as a high activity of 
phosphatases, especially alkaline phosphatase (AP) [10]. AP-mediated phosphoester hydrolysis may facilitate mineralization by several possible mechanisms, including augmentation of regional phosphate concentrations and degradation of endogenous inhibitors of calcification, such as pyrophosphate and phosphocitrate. Matrix vesicle-like cell fragments have been noted in several pathological calcification processes, including calcific degeneration of aged aortic valves [11] and mineralization in atherosclerotic plaque [12].

\section{Role of alkaline phosphatase in bioprosthetic tissue mineralization}

Since BPT mineralization, other pathological cardiovascular calcifications, and physiological mineralization are largely initiated in cell-derived membranous structures, and since AP likely has an important function in mineral nucleation in bone, we hypothesized that AP might contribute to bioprosthetic tissue mineralization. Despite glutaraldehyde crosslinking, bovine pericardium retains histochemically-demonstrable AP hydrolytic activity localized to the membranous cell remnants known to be the initial sites of mineralization [13]. Moreover, there is histochemically-demonstrable AP in 1) bovine pericardium pretreated in glutaraldehyde for as long as $\mathbf{8 2}$ days, albeit diminished in intensity, 2) fresh porcine aortic valve, and 3) glutaraldehyde-pretreated porcine valve implanted 5 months in a sheep [14]. However, although AP activity is reproducibly extractable from fresh bovine pericardium (30.4 $\pm 6.8 \mathrm{~nm} \mathrm{pnpp} / \mathrm{min} / \mathrm{mg}$ protein), it cannot be detected chemically after glutaraldehyde fixation for as little as 24 hours [14].

Following subQ implantation of BPT into rats, AP enzymatic activity accumulates rapidly within 48-72 hours. Extractable AP activity rises sharply to a peak at 72 hours, concurrent with the onset of calcification. It then declines to near baseline levels by 21 days. Mineral deposition progresses to maximal at 120 days, while AP activity is at a reduced level. Thus, maximal AP activity correlates with the onset of mineralization, but calcification progresses concurrent with stable or falling AP levels.

\section{Table 1}

Correlation of chemical and morphological assays for alkaline phosphatase in subcutaneously implanted bioprosthetic tissue in rats

\begin{tabular}{cccc}
\hline Time (d) & AP (Enz Units) & AP (Cyto) & $\mathrm{Ca}^{++}(\mu \mathrm{g} / \mathrm{mg})$ \\
\hline 0 & 0 & ++ & $1.1+1-0.1$ \\
1 & $107+/-10$ & +++ & $1.3+1-0.4$ \\
3 & $424+1-67$ & ++++ & $2.2+1-0.3$ \\
21 & $96.8+1-6.9$ & + & $56.8+1-3.1$ \\
120 & $52.4+1-2.7$ & ND & $239+1-20$ \\
\hline
\end{tabular}

Mean \pm SEM $;+=$ mild,$++=$ moderate, $+++=$ prominent,$++++=$ most prominent; $\mathrm{ND}=$ not done; Reprinted by permission from Reference 14 . 
Histochemical studies demonstrate intense AP activity adsorbed into and near the surface of the BPT in 24-72 hour explants, predominately associated with host inflammatory cells adhering to the surface of the implant, but not with pre-existing intrinsic cuspal celis. Histochemically-demonstrable AP is also qualitatively much less intense, though visible at the cuspal surface, at 21 days following implantation, at which time AP activity has declined. Correlation of chemical and morphological results is summarized in the Table [14].

Extractable AP activity was markedly reduced by preincubation of bovine pericardium in either $\mathrm{AlCl}_{3}$ or $\mathrm{FeCl}_{3}$ at concentrations $(0.1 \mathrm{M})$ sufficient to suppress calcification [14]. The extent of inhibition of calcification is comparable for incubations in $0.1 \mathrm{M} \mathrm{FeCl}_{3}$ and $0.1 \mathrm{M} \mathrm{AlCl}_{3}$. Reduction in extractible AP activity and inhibition of calcification correlates with loss of histochemically-demonstrable enzyme activity. More dilute preincubations in either $10^{-5} \mathrm{M} \mathrm{AlCl}_{3}$ or $10^{-5} \mathrm{M} \mathrm{FeCl}_{3}$ do not significantly inhibit calcification, but do mildly reduce AP activity.

\section{Comment}

Our studies on AP in BPT mineralization have two primary results: 1) simultaneous to the onset of mineralization of bioprosthetic tissue, AP content sharply increases, with enhanced activity localized to the tissue surface, probably associated with inflammatory cells, and 2) intrinsic $A P$ is neutralized by incubations of $\mathrm{BPT}$ in $\mathrm{AlCl}_{3}$ and $\mathrm{FeCl}_{3}$, treatments that markedly reduce mineralization.

The data suggest that the intrinsic AP activity associated with devitalized BPT cells is augmented by extrinsic AP from the recipient. This results in a marked increase in AP activity in the first $\mathbf{7 2}$ hours of BPT rat subdermal implants, during which calcification is initiated. Onset of calcification could involve both intrinsic AP activity associated with the site of the initial calcific deposits, and absorbed extrinsic AP that could accrue from inflammatory cells or serum, or both. Adsorbed AP could serve to provide a rapid rise in regional internal phosphate concentration sufficient to trigger mineral formation in devitalized cells. This is analogous to physiological mineralization in which matrix vesicle calcification occurs in synchrony with a peak in AP activity, that then declines as bulk mineralization progresses [15]. This implies a mechanistic role for AP in early mineral nucleation in BPT similar to that in matrix vesicles of physiological calcification.

Preincubation in either $\mathrm{FeCl}_{3}$ and $\mathrm{AlCl}_{3}$ inhibits BPT calcification, associated with reduced $A P$ activity. $A l$ and Fe may act to interfere with $A P$ and perhaps its contribution to mineralization by multiple independent mechanisms. For example, $A l$ and Fe preincubations would be predicted to interfere with intrinsic AP activity localized to devitalized cells, and also interfere with uptake, thereby perhaps blunting or eliminating the early rise in AP activity.

Is AP relevant to clinical bioprosthetic valve calcification? Further studies are required to definitively answer this question. Moreover, absorption of extrinsic AP might be important to calcification of other tyjes of biomaterials since this enzyme could be readily adsorbed on and absorbed into many implantable biomaterials. 
Calcification of polyurethane in valves, artificial hearts and ventricular assist systems usually occurs as deposition of calcium phosphates in devitalized cells and their debris, and perhaps adherent blood platelets, that also contain high AP activity.

Strategies for preventing biomaterial calcification could be based on AP inhibition. Calcification inhibitors described previously for bioprosthetic valves [1] might act in part through effects on AP-dependent mechanisms. For example, sodium dodecyl sulfate (SDS), may act by removing part or all of the intrinsic AP present in BPT, rendering it temporarily less calcifiable until additional absorption can take place. Likewise, diphosphonates inhibit BPT calcification, and also inhibit AP; diphosphonates are structurally related to pyrophosphate, a well known physiologic inhibitor of calcification and also an AP substrate, whose anticalcification potential is neutralized by AP.

\section{References}

1 Schoen FJ. In: Cardiovascular Pathology, 2nd Ed., Silver MD (ed), Churchill Livingstone 1991, p. 1547.

2 Schoen FJ, Harasaki H, Kim KM, et al. J Biomed Mater Res: Applied Biomater 1988;22, A11.

3 Levy RJ, Schoen FJ, Levy JT, et al. Am J Pathol 1983;113:143.

4 Levy RJ, Schoen FJ, Howard SL. Am J Cardiol 1э83;52:629.

5 Golomb G, Schoen FJ, Smith MS, et al. Am J Pathol 1987;127:122.

6 Schoen FJ, Levy RJ, Nelson AC, et al. Lab Invest 1985;52:523.

7 Schoen FJ, Tsao JW, Levy RJ. Am J Pathol 1986;123:143.

8 Webb CL, Schoen FJ, Alfrey AC, et al. Am J Pathol 1991.

9 Levy RJ, Schoen FJ, Sherman FS, et al. Am J Pathol 1986;122: 71.

10 Anderson HC. Lab Invest 1989;60:320.

11 Kim KM, Valigorsky JM, Mergner WJ, et al. Hum Pathol 1976;7:47.

12 Tanimura A, McGregor DH and Anderson HC. Proc Soc Exp Biol Med 1983;172:173.

13 Maranto AR, Schoen FJ. Circ Res 1988;63:844.

14 Levy RJ, Schoen FJ, et al. J Biomed Mater Res 1991;25:905.

15 Eenge BR, Sauer GR, Wu LNY, et al. J Biol Chem 1988;263:18513. 